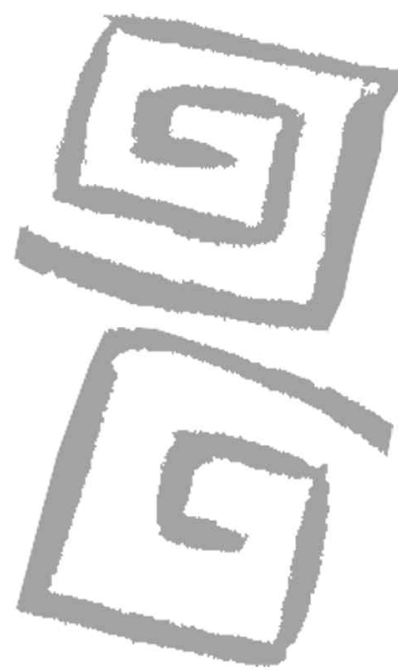

\title{
¿Aceptación o rechazo de la estandarización clínica? Médicos chilenos hablan de las guías clínicas y canastas de prestaciones
}

\author{
Acceptance or rejection of clinical standardization? \\ Chilean doctors discuss clinical practice guidelines and \\ benefit packages
}

Lemp, Sebastián'; Calvo, Esteban²

${ }^{1}$ Sociólogo. Magíster en Sociología. Academia Diplomática Andrés Bello, Chile.

slemp@minrel.gov.cl

${ }^{2}$ Sociólogo. Doctor en Sociología. Profesor e Investigador del Instituto de Políticas Públicas, Facultad de Economía y Empresa, Universidad Diego Portales, Chile. esteban.calvo@udp.cl
RESUMEN Este estudio analiza la aceptación de los médicos respecto de la estandarización de la atención clínica que conlleva la reforma de Acceso Universal con Garantías Explícitas (AUGE) y de Garantías Explícitas en Salud (GES) iniciada en el año 2005 en Chile. Se realizaron 18 entrevistas semiestructuradas, en las que se exploran cuatro hipótesis vinculadas al nivel de aceptación de la estandarización y su variación según los años de práctica clínica, el tipo de instrumento (guía clínica o canasta de prestaciones) y la especialidad (médica o quirúrgica). Los resultados sugieren que no existe un rechazo generalizado hacia la estandarización de los procesos clínicos, sino importantes diferencias en el discurso de los médicos. El grado de aceptación depende tanto de los años de experiencia clínica como del tipo de instrumento evaluado. Se discute la implicancia de estos resultados para diseñar e implementar reformas de salud exitosas, que consideren la racionalidad de la profesión médica y su énfasis en la discrecionalidad individual, variabilidad terapéutica y capacidad para adecuarse a las circunstancias particulares de su práctica.

PALABRAS CLAVE Práctica Profesional; Autonomía Profesional; Actitud del Personal de Salud; Guía de Práctica Clínica; Control de Costos; Chile.

ABSTRACT This study analyzes the degree to which Chilean doctors accept the standardization of clinical health care associated with the 2005 Health Reform AUGEGES (from the Spanish Acceso Universal con Garantías Explícitas - Garantías Explícitas en Salud). Using 18 semi-structured interviews, four hypotheses were explored in relation to the level of acceptance of standardization and its variation according to years of clinical experience, the type of instrument (clinical practice guidelines or benefit packages), and the specialty (medical or surgical). Rather than a generalized rejection of the standardization of clinical procedures, the results suggest important differences within the discourse of the doctors. The level of acceptance depends both on years of clinical experience and the type of instrument evaluated. We discuss the implications of these results for the design and implementation of successful health reforms, incorporating the rationale of the medical profession and its emphasis on individual discretion, variability of treatment decisions, and the ability to adjust to the particular circumstances of the practice.

KEY WORDS Professional Practice; Professional Autonomy; Attitude of Health Personnel; Practice Guidelines; Cost Control; Chile. 


\section{INTRODUCCIÓN}

El uso creciente de guías clínicas y canastas de prestaciones para estandarizar la práctica médica constituye un cambio substancial en el proceso de salud-enfermedad-atencióncuidado (PSEAC). Este cambio ha sido ampliamente discutido en las ciencias sociales y las ciencias de la salud y muchas veces se lo ha descrito como un aumento de la racionalización científico-técnica en las prácticas de salud (1). Sin embargo, la evidencia disponible sobre el punto de vista de los médicos acerca de esta estandarización es limitada (2). Este estudio pretende contribuir al debate teórico acerca de la estandarización clínica mediante el análisis de las reacciones discursivas de los médicos frente a la estandarización de su práctica. También pretende ser un aporte para guiar los procesos de reforma en la atención en salud que involucren una mayor estandarización clínica. Específicamente, este estudio explora el grado de aceptación que reportan los médicos chilenos respecto de la estandarización clínica introducida con las guías clínicas y las canastas de prestaciones que son parte de la reforma de Acceso Universal con Garantías Explícitas (AUGE) y de Garantías Explícitas en Salud (GES).

La reforma AUGE-GES, iniciada en el año 2005 en Chile e inspirada en el enfoque de los derechos sociales en salud, consiste en que las instituciones encargadas de la salud de la población deben respetar una normativa legal y administrativa inspirada en el derecho internacional (3). Esta reforma global del sistema de salud chileno prioriza los recursos de atención a ciertas patologías (en 2011 sumaban 69) escogidas a partir de consideraciones sobre prevalencia, incidencia, morbilidad, invalidez y costos. La reforma incorpora además guías clínicas que determinan los tiempos máximos de espera para obtener una atención y canastas de prestaciones que detaIlan los medicamentos y tratamientos cubiertos para cada patología asegurada. Al certificar la atención de un paciente afectado por una patología asegurada, el Fondo Nacional de Salud (FONASA) le transfiere al prestador, sea público o privado, los recursos definidos por una prima universal. En síntesis, la reforma asegura a indivi- duos enfermos de patologías específicas, cuatro garantías: acceso, oportunidad, financiamiento y calidad (4).

La garantía de calidad es particularmente importante para este estudio porque exige el cumplimiento de estándares mínimos a los establecimientos de salud, pero no será exigida legalmente hasta que se termine la acreditación de los prestadores. Al momento de exigir legalmente esta garantía será especialmente valioso comprender la aceptación de la estandarización clínica por parte de los médicos. Sin embargo, las evaluaciones de la reforma realizadas a la fecha han prestado poca atención a los efectos de la estandarización clínica y se han concentrado en las listas de espera y la efectividad de las prestaciones (5-7).

Estudios realizados en países anglosajones indican que el grado de aceptación de la estandarización por parte de los médicos es generalizadamente bajo (8-10). En estas investigaciones, los médicos reportan estar en desacuerdo con la evidencia utilizada en el diseño de las guías clínicas y canastas de prestaciones, disconformes con la rigidez o simplicidad de su diseño y dudosos respecto al riesgo de inducir malas prácticas (8).

La sociología médica ha intentado explicar esta baja aceptación de la estandarización argumentando que la práctica profesional médica responde a una concepción de autonomía, autorregulación, responsabilidad personal y racionalidad práctica (11-14). Los médicos tienden a seguir sugerencias solo si coinciden con su propio criterio, se resisten a cambiar su práctica clínica sobre la base de consideraciones abstractas y fundan su criterio clínico en una racionalidad práctica que destaca la importancia de ajustar dosis, tiempo e intensidad hasta encontrar el óptimo para cada paciente en particular. En general, priorizan un criterio clínico complejo y flexible que permita correr riesgos, a diferencia de seguir un conjunto de rutinas estandarizadas (13). Tomando en cuenta estos antecedentes teóricos, se plantearon cuatro hipótesis vinculadas al nivel de aceptación de la estandarización y su variación según los años de práctica clínica, el tipo de instrumento (guía clínica o canasta de prestaciones) y la especialidad (médica o quirúrgica): 
1. El grado de aceptación de la estandarización es generalizadamente bajo entre los médicos, quienes evalúan los instrumentos de estandarización a partir de un criterio clínico ajeno a la rutinización y a una normativa rígida de tratamientos.

2. Los médicos pertenecientes a las últimas cohortes de egresados y con menor experiencia clínica, reportan una mayor aceptación de la estandarización del quehacer médico.

3. Las guías clínicas AUGE-GES cuentan con mayor aceptación que las canastas de prestaciones ya que las guías fueron diseñadas con la participación de médicos y por lo tanto responden de forma más directa al criterio de expertos y a la evidencia científica. Por el contrario, se espera que las canastas de prestaciones generen una menor aceptación de la comunidad médica ya que estandarizan los costos de la atención de acuerdo a criterios económicos y no necesariamente a criterios médicos (15).

4. Las especialidades quirúrgicas reportan mayor aceptación de la estandarización, en tanto enfrentan patologías más localizadas donde la sanación se lleva principalmente en el hospital, mientras que las especialidades médicas deben atender patologías crónicas y multisistémicas donde el médico debe regularmente ajustar las dosis de tratamiento de acuerdo al paciente (16).

Las cuatro hipótesis en su conjunto exploran el grado y variabilidad de aceptación de la estandarización clínica expresada por los médicos.

\section{MATERIAL Y MÉTODO}

Para explorar empíricamente las cuatro hipótesis de este estudio se utilizó una metodología cualitativa de entrevistas semiestructuradas que permiten captar tanto experiencias y significados individuales como discursos que remiten a significados sociales del entorno, tales como prácticas y criterios compartidos por el grupo social con el que el entrevistado se identifica (17).
Se realizaron 14 entrevistas a médicos y cuatro a funcionarios administrativos (médico, enfermera, secretario e ingeniero). Aunque el foco del estudio son los médicos practicantes, los funcionarios administrativos evalúan rutinariamente la aplicación de guías clínicas y canastas de prestaciones, por lo tanto, la información entregada por los administrativos permite mejorar la pauta de entrevista aplicada a los médicos y explorar la validez externa de sus opiniones mediante la triangulación de datos (18).

El muestreo inicial fue de tipo intencionado o teórico y mediante un sistema de bola de nieve o avalancha se expandió a los contactos de los mismos entrevistados. Se seleccionaron solamente entrevistados que tuvieran experiencia laboral en la utilización de guías y canastas, y que trabajaran en hospitales públicos del sector Oriente, Sur-oriente y Norte de la ciudad de Santiago. El tamaño muestral se fijó balanceando un criterio de saturación teórica, según el cual no es necesario realizar una nueva entrevista cuando ya no agrega información significativa, con un criterio de máxima heterogeneidad que implica segmentar la muestra por especialidades médicas (cardiología, neurología, neonatología y reumatología) y quirúrgicas (neurocirugía, traumatología y urología), y por años de experiencia (menos de 15 y 15 o más años) (19).

Todas las entrevistas fueron aplicadas por uno de los autores, entre el 18 de octubre y el 10 de diciembre del año 2010, en la privacidad de oficinas o boxes de los hospitales, durante el tiempo libre de los entrevistados. Al comenzar cada entrevista se explicó a los participantes el tema de la investigación, se les aseguró confidencialidad y se obtuvo su consentimiento informado para proseguir con la aplicación de una pauta de entrevista con preguntas orientadoras. La pauta (disponible por solicitud) buscaba conocer las opiniones respecto a la estandarización de la atención clínica y se organizó en cuatro temas: guías clínicas y protocolos, guías clínicas AUGE-GES, canastas de prestaciones AUGEGES y consecuencias de la reforma AUGE-GES. La profundidad obtenida en cada tema dependió de las experiencias y reacciones de los entrevistados frente a las preguntas orientadoras, resultando en entrevistas con un rango de duración de 30 a 60 minutos. 
Todas las entrevistas fueron transcritas y las opiniones relevantes fueron introducidas como citas textuales a una tabla dividida en secciones correspondientes a los temas de la pauta de entrevista y a temas emergentes. Siguiendo los principios de la teoría fundamentada, utilizamos estos datos para reelaborar conceptos teóricos y explorar el poder explicativo de cada hipótesis (20). La siguiente sección resume los resultados obtenidos mediante citas ilustrativas y una ilustración gráfica de las tendencias en las opiniones de los entrevistados.

\section{RESULTADOS}

La opinión de cada entrevistado fue clasificada en una jerarquía cualitativa simple, como apoyando o contradiciendo cada hipótesis, o como neutral en caso de ambigüedad o falta de opinión. Esta información permitió calcular el número total de opiniones que apoyan, son neutrales o contradicen cada hipótesis. La Figura 1 resume estos resultados y utiliza esferas de distintos tamaños para ilustrar la distribución de opiniones cualitativas según su grado de apoyo a cada hipótesis.

En el eje superior de la Figura 1, se observa que los resultados del análisis de las entrevistas son contradictorios con la hipótesis sobre un grado de aceptación de la estandarización generalizadamente bajo. Existe un discurso de baja aceptación junto a otro de mayor aceptación. El primer discurso enfatiza que la "medicina es un arte" que debe considerar simultáneamente al paciente y las mejores prácticas en medicina. Este discurso reconoce la importancia del uso de las mejores prácticas en medicina, pero enfatiza que la práctica del médico no se corresponde con una mecánica de asociación unívoca entre enfermedad y cura.

\begin{abstract}
Hace algún tiempo ya se han comenzado a realizar estas guías, y la tendencia es ir aumentando... las guías... los manejos de pacientes protocolizados... pero eso le quita un poco al "arte de la medicina", entonces por qué, o si no se va transformar todo en... identificar una enfermedad y hacerla calzar con un diagnóstico y aplicar esa guía, esa norma ya... y la medicina no es tan así... como te digo hay mucha variabilidad personal de distintas enfermedades que pudiera tener... (Especialidad Médica, 15 o más años de experiencia)
\end{abstract}

El discurso con mayor aceptación enfatiza la importancia de una estandarización clínica que incorpore criterios de "flexibilidad e innovación terapéutica".

Figura 1. Distribución del total de opiniones de los entrevistados según el grado de apoyo para cada hipótesis. Santiago de Chile, 2010.

Hipótesis
- Nivel: bajo nivel de aceptación de
la estandarización
- Experiencia: a mayor experiencia
menor aceptación
- Instrumento: mayor aceptación
para guías clínicas que canastas
especialidad: mayor aceptación
para médicas que quirúrgicas

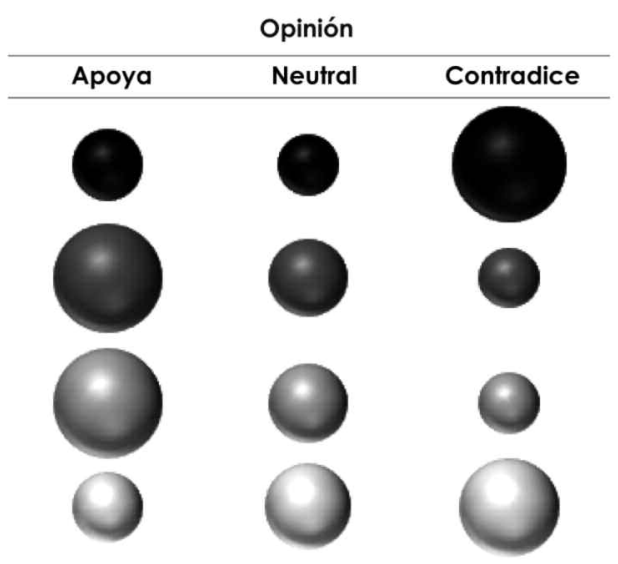

Fuente: Elaboración propia. 
Las guías nos van un poco guiando, valga la redundancia, en el quehacer de la patología del paciente, y es por eso que cuando uno confecciona una guía clínica, lo ideal es que sea lo más... tratar de encauzar bien la patología, en cuanto a... en el eje en cuanto al diagnóstico y al tratamiento, pero siempre dejando ventanas abiertas de variación que uno pueda hacer sobre el manejo específico del paciente. (Especialidad Quirúrgica, menos de 15 años de experiencia)

El segundo eje horizontal de la Figura 1 muestra un fuerte apoyo a la segunda hipótesis de este trabajo, la cual postula que el grado de aceptación de la estandarización depende de los años de experiencia clínica. Los resultados sugieren una combinación de efectos de edad y cohorte. En particular, una menor cantidad de años de práctica clínica y el haber sido formado como especialista utilizando guías clínicas de Europa y Estados Unidos se asocian con una mayor aceptación de la estandarización.

En el fondo, la idea es que todos los traumatólogos del servicio estén al tanto de qué consiste la guía, y la conozcan, e idealmente ojalá todos las lean y sepan de qué se trata, pero no es fácil sobre todo cuando hay traumatólogos con... con más años de experiencia, algunos la van a aplicar, otros no, dependiendo de cómo haya sido su realidad, su experiencia, cómo le haya ido... (Especialidad Quirúrgica, menos de 15 años de experiencia)

La salud y los enfermos no son rígidos. Las evoluciones de las enfermedades no son rígidas. No siempre se presentan por... como debiera de ser... de presentarse, siempre pueden... una enfermedad puede tener mil manifestaciones que pueden hacer que se constituya, y a veces no es tan así... Así no se puede rigidizar algo que no es rígido, no puede rigidizar... puedes ponerla dentro de ciertos márgenes, pero no puedes... tratar de ponerle límites concretos. (Especialidad Médica, 15 años o más de experiencia)

El tercer eje horizontal de la Figura 1 también sugiere que la opinión de los entrevistados tiende a apoyar la tercera hipótesis. Específicamente, los resultados sugieren que las guías clínicas son más aceptadas que las canastas de prestaciones. Un grupo importante de entrevistados, sin diferencias por edad o especialidad, sostiene que las guías clínicas han significado un mejor acceso y oportunidad en la atención de pacientes.

Pero más bien... lo que yo veo en esta reforma es que se ha mejorado evidentemente el momento del diagnóstico de enfermedades graves y lo más oportuno que ha sido el tratamiento de estas enfermedades graves. Eso es un avance muy importante que logró el AUGE en algunas enfermedades. (Especialidad Médica, 15 años o más de experiencia)

A pesar de existir un discurso favorable hacia la estandarización introducida por las guías clínicas, estas también son criticadas por poseer alternativas restringidas de tratamiento que reflejan criterios de restricción de recursos antes que una mejor atención.

\begin{abstract}
A ver... uno le gustaría siempre que fuera más amplia, por ejemplo en los pacientes artríticos, que es donde tenemos GES nosotros, y los artrósicos, no está contemplado, en los artrósicos sobretodo, no está contemplado el reemplazo articular, las prótesis. Ni siquiera la posibilidad de que accedan a una cirugía, no está contemplado en las guías GES [...] En realidad uno se siente ahí frustrado, porque no son completas, no es lo que uno quisiera, no es lo que realmente el enfermo podría llegar a necesitar. (Especialidad Médica, 15 años o más de experiencia)
\end{abstract}

La estandarización de la práctica médica que conlleva restricciones de recursos es claramente controversial entre los médicos y genera todavía más rechazo al momento de evaluar las canastas de prestaciones. Las canastas son criticadas por no financiar los mejores medicamentos, no ajustarse al costo real de los prestadores y no considerar complicaciones.

La disposición de las canastas ha puesto un piso, cierto, de qué cosas se tienen que usar. Pero ese piso tiene que ser mejorado [...] No importa que [el medicamento] sea barato, pero que sea bueno. 
Lo malo es que sea el barato y de que haya evidencia de que no sea el mejor. Eso es lo cuestionable. Es un problema ético... Uno no lo usaría... ni en uno mismo, o en el familiar de uno... (Especialidad Médica, 15 años o más de experiencia)

El eje horizontal inferior de la Figura 1 ilustra que las opiniones de los entrevistados contradicen la cuarta hipótesis de este trabajo. Específicamente, las especialidades quirúrgicas no parecieran ser más susceptibles de estandarizar que las especialidades médicas (citas anteriores reflejan la opinión de especialidades médicas). Ciertamente las especialidades quirúrgicas se enfrentan a patologías más localizadas que las especialidades médicas y realizan el proceso de sanación con mucha más frecuencia en el hospital, pero los médicos cirujanos también confían ampliamente en su propio criterio y además se especializan en técnicas quirúrgicas particulares que ajustan a su experiencia, a las circunstancias del paciente y a los recursos del contexto laboral.

Porque cuando uno hace las guías, las hace sobre un pool de pacientes, sobre la estadística de los pacientes, de cómo van a andar con ciertas drogas, con ciertos procedimientos, y hay pacientes que pueden escapar a eso, a ese tipo de resultados, es por eso que uno tiene que tener ventanas abiertas en cuanto al uso de las guías para poder tratar ese tipo de variaciones de los pacientes. (Especialidad Quirúrgica, menos de 15 años de experiencia)

...considerando el ambiente de los médicos, en el sentido de que somos bien diversos y cada uno bien poderoso con su opinión, casi... casi altaneros muchas veces con la opinión personal, entonces una guía que podamos aplicar todos, es una guía bastante difícil de confeccionar [...] Como guía yo creo que es bueno, pero... cuando termina aplicándose al pie de la letra, de repente es un poco peligroso, y además poco... entretenido profesional y académicamente. (Especialidad Quirúrgica, 15 años o más de experiencia)

Todos estos resultados se mantienen consistentes al explorar el discurso de los profesionales administrativos incluidos en la muestra, lo cual sugiere validez externa de los resultados.

\section{DISCUSIÓN}

Este estudio utilizó entrevistas semiestructuradas para explorar el grado de aceptación que los médicos reportan respecto de la estandarización de la atención clínica que conlleva la reforma AUGE-GES. Los resultados sugieren que no existe un rechazo generalizado hacia la estandarización, sino importantes diferencias en el discurso de los médicos, habiendo grupos que enfatizan los beneficios y otros las dificultades.

Los resultados también sugieren que el grado de aceptación de la estandarización depende de los años de experiencia y del tipo de instrumento. El grado de aceptación es mayor entre médicos con menos experiencia y formación más reciente, ya que tienden a confiar menos en su propio criterio y a estar más familiarizados con el uso de instrumentos de estandarización en otros países que los médicos de mayor edad y experiencia. La formación continua aparece en este contexto como un aspecto clave para el éxito sostenido de la reforma AUGE-GES y otras reformas con un componente de estandarización clínica, especialmente si se toma en cuenta que los médicos jóvenes formados en la nueva medicina podrían disminuir su grado de aceptación a medida que acumulen experiencia.

En cuanto a la variación en la aceptación de la estandarización por tipo de instrumento, los resultados sugieren que las guías clínicas generan mayor aceptación que las canastas de prestaciones. En general, las guías clínicas son percibidas como flexibles y adecuadas, aunque en varios casos el profesional hubiese esperado mayor oferta y vigencia de alternativas terapéuticas. Un desafío no resuelto de la reforma AUGEGES es determinar un orden de jerarquía entre las indicaciones de las canastas de prestaciones. Como las canastas contienen el detalle del medicamento y del tratamiento que será efectivamente financiado, cada vez que el médico decide realizar un tratamiento no contemplado, se produce una diferencia entre el costo real y el dinero transferido por las aseguradoras públicas o privadas, hecho que hace que la opinión de los médicos se torne más crítica respecto al uso de las canastas.

En su conjunto, los resultados de este estudio destacan la importancia que le asignan 
los médicos al hecho de escoger entre diferentes alternativas terapéuticas y adecuarse a las circunstancias particulares de los pacientes y al contexto de su práctica médica (21). Esta racionalidad de la profesión médica tiene una relación dialéctica con el uso creciente de evidencia científica en medicina. La relación dialéctica implica una tensión entre tesis y antítesis, que en este caso ha sido descrita como una lucha paradigmática entre el enfoque tradicional fisiopatológico orientado al individuo y el enfoque epidemiológico orientado a poblaciones (22). La relación dialéctica también implica una síntesis, que en este caso ha sido descrita como una falsa dicotomía entre el enfoque fisiopatológico y epidemiológico, ya que ambos deben considerar simultáneamente factores individuales y poblacionales, están determinados por el contexto (histórico, político, económico, social, cultural e institucional) y encuentran unidad en la propia subjetividad del médico (23-25). Reconocer esta relación dialéctica y su influencia en el PSEAC al momento de diseñar e implementar reformas de salud puede contribuir favorablemente a su éxito.

Los resultados de este estudio tienen importantes implicancias prácticas para guiar los esfuerzos de la reforma AUGE-GES porque esta ha significado una reorganización nacional de la atención en salud, buscando mejorar los indicadores epidemiológicos y la satisfacción usuaria, al mismo tiempo que introduce herramientas de estandarización del quehacer médico. Una vez terminado el proceso de acreditación de los prestadores, la reforma AUGE-GES contempla la posibilidad de exigir legalmente el cumplimiento de estándares de calidad que, sumado al efecto de la estandarización ya introducida mediante guías clínicas y canastas de prestaciones, probablemente afectarán de manera importante la discrecionalidad clínica.

A partir de los resultados aquí presentados, estudios futuros podrían utilizar encuestas y muestras representativas para analizar el tema con métodos cuantitativos y extender la investigación, por ejemplo, para considerar las diferencias en el grado de aceptación de la estandarización dependiendo del rol de los médicos dentro de su profesión. La variabilidad encontrada en este estudio es substancial, pero podría haber sido subestimada al no incluir médicos académicos ni médicos generales con fuerte carga administrativa. Estimar con precisión la variabilidad en la aceptación de la estandarización clínica permitirá guiar de forma más eficiente los esfuerzos de reforma al sistema de salud en Chile y aportar a la discusión global sobre las consecuencias de la estandarización del quehacer médico.

\section{AGRADECIMIENTOS}

Los resultados y conclusiones de esta investigación son de los autores y no pretenden representar la visión de la Academia Diplomática "Andrés Bello" o la Universidad Diego Portales. Los autores agradecen a Ariel Azar, Francisca Florenzano, Vicente Montenegro, Melchor Lemp, Nicolás Rodríguez, Nicolás Somma, Eduardo Valenzuela y dos evaluadores anónimos por sus comentarios y sugerencias a versiones anteriores de este artículo. Sin embargo, los autores son los únicos responsables de errores u omisiones.

\section{REFERENCIAS BIBLIOGRÁFICAS}

1. De Carvalho JR. Para comprender el sentido práctico de las acciones de salud: Contribuciones de la hermenéutica filosófica. Salud Colectiva. 2008;4(2):159-172.

2. Rashidian A, Eccles M, Russell I. Falling on stony ground? A qualitative study of implementation of clinical guidelines' prescribing recommendations in primary care. Health Policy. 2008;85(2):148-161.
3. Ase I, Burijovich J. La estrategia de Atención Primaria de la Salud: ¿progresividad o regresividad en el derecho a la salud? Salud Colectiva. 2009;5(1):27-47.

4. Sánchez H. Implicancias del AUGE en medicina y cirugía: Ley de Garantías Explícitas en Salud. Revista Chilena de Cirugía. 2006;58(6):397-399.

5. González F. La implementación del Plan de Acceso Universal y Garantías Explícitas (Plan AUGE) en pacientes con insuficiencia renal crónica genera mayores listas de espera entre todos 
los usuarios. Revista Médica de Chile. 2003;134(10):545-551.

6. Andia M, Gederlini A, Ferreccio C. Cáncer de vesícula biliar: Tendencia y distribución del riesgo en Chile. Revista Médica de Chile. 2006;134(5):565-574.

7. González F. La implementación del Plan de Acceso Universal y Garantías Explícitas (Plan AUGE) ha deteriorado la calidad del tratamiento de pacientes con insuficiencia renal terminal. Revista Médica de Chile. 2006;134(10):1288-1294.

8. Cabana MD, Rand CS, Powe NR, Wu AW, Wilson $\mathrm{MH}$, Abboud PA, et al. Why don't physicians follow clinical practice guidelines? A framework for improvement. Journal of the American Medical Association. 1999; 282(15):1458-1467.

9. Farquhar CM, Kofa EW, Slutsky JR. Clinicians' attitudes to clinical practice guidelines: A systematic review. The Medical Journal of Australia. 2002;177(9):502-506.

10. De Jong J, Groenewegen P, Spreeuwenberg $P$, Schellevis F. Do guidelines create uniformity in medical care? Social Science and Medicine. 2010;70(2):209-216.

11. Parsons T. El sistema social. Madrid: Alianza Editorial; 1988.

12. Armstrong D. Clinical autonomy, individual and collective: the problem of changing doctors' behaviour. Social Science and Medicine. 2002;55(10):1771-1777.

13. Coe R. Sociología de la Medicina. Madrid: Alianza Editorial; 1984.

14. Suchman MC, Dimick MD. A profession of IT's own: The rise of health information professionals in american healthcare. En: Rothman DJ, Blumenthal D. Medical Professionalism in the New Information Age. New Brunswic: Rutgers University Press; 2010.

15. Lenz R. Proceso político de la reforma AUGE de salud en Chile: Algunas lecciones para América Latina. Una mirada desde la economía política. Santiago de Chile: CIEPLAN; 2007. (Serie de Estudios Socio/Económicos N³8).

16. Moret-Hartman $M$, Knoester $P$, Hekster $Y$, van der Wilt G. Non-compliance on the part of the professional community with a national guideline: An argumentative policy analysis. Health Policy. 2006;78(2-3):353-359.

17. Merlinsky G. La entrevista como forma de conocimiento y como texto negociado. Cinta de Moebio. 2006;27:27-33.

18. Cisterna F. Categorización y triangulación como procesos de validación del conocimiento en investigación cualitativa. Theoria. 2005;14(1):61-71.

19. Osses Bustingorry S, Sánchez Tapia I, Ibáñez Mansilla F. Investigación cualitativa en educación. Hacia la generación de teoría a través del proceso analítico. Estudios Pedagógicos. 2006;32(1):119-133.

20. Strauss A, Corbin J. Bases de la investigación cualitativa: Técnicas y procedimientos para desarrollar la teoría fundamentada. Bogotá: CONTUS, Editorial Universidad de Antioquia; 2002.

21. Mottur-Pilson C, Snow V, Bartlett K. Physician explanations for failing to comply with "best practices". Effective Clinical Practice. 2001;4(5):207-213.

22. Timmermans S, Kolker ES. Evidence-based medicine and the reconfiguration of medical knowledge. Journal of Health and Social Behavior. 2004;45(Extra Issue):177-193.

23. Duarte Nunez E. Por una epistemología de la Salud Colectiva: los conceptos sociológicos. Salud Colectiva. 2010;6(1):11-19.

24. Diez Roux AV. En defensa de una epidemiología con números. Salud Colectiva. 2007;3(2):117119.

25. Almeida Filho N. For a general theory of health: preliminary epistemological and anthropological notes. Cadernos de Saúde Pública. 2001;17(4):753-770.

\section{FORMA DE CITAR}

Lemp S, Calvo E. ¿Aceptación o rechazo de la estandarización clínica? Médicos chilenos hablan de las guías clínicas y canastas de prestaciones. Salud Colectiva. 2012;8(1):61-68. 